\title{
La materialidad del encuentro. Una indagación de la impression management en entornos organizacionales desde la materialidad de la interacción
}

\author{
Fran Morente \\ Universitat de Vic \\ fjmp.1984@gmail.com
}

Resumen: Más allá del utilitarismo, las organizaciones son un entorno fértil para la sociología. El concepto impression management ha ahondado en esta complejidad, pues evidencia las tensiones dramatúrgicas de los agentes. A pesar de las diversisimas investigaciones con este concepto, se ha obviado la materialidad de las interacciones. Así, nuestro objetivo es investigar la impression management desde múltiples materialidades cotidianas, para entender el self — ser, apariencia-y generar teoría que aporte conocimiento sobre la socialización organizacional. Con base en el diseño grounded theory sobre siete casos, desgranamos la impression management en e-mails, chats, whatsapps, llamadas telefónicas, videoconferencias, reuniones y desayunos, $y$, finalmente, definimos el concepto de «ixtimidad» que contribuye a entender cómo los agentes luchan por proyectar sus imágenes en los otros.

Palabras clave: Goffman; organización; materialidad; impression management; Grounded Theory 
The materiality of the encounter. An investigation into impression management in organizational environments from the perspective of the materiality of interactions

Abstract: Beyond utilitarianism, organizations are a fertile milieu for sociology. The concept of impression management has deepened this complexity as it demonstrates the agents' dramaturgical tensions. In spite of the very diverse research into this concept, the different materialities of interaction have been ignored. Thus, our aim is to research impression management from multiple everyday materialities, to understand the self (being, appearance) and to generate a theory which contributes new knowledge on organizational socialization. The study uses the grounded theory method to investigate seven cases in which we describe impression management in e-mails, chats, Whatsapps, telephone calls, videoconferences, meetings and breaks and, finally, we define the concept 'ixtimacy' to help our understanding of how agents struggle to project their images onto others.

Keywords: Goffman; Organization; Materiality; Impression Management; Grounded Theory 


\section{Marco y objetivo}

Tomándolo de Goffman, el concepto central de este artículo es la impression management (en adelante, IM), y su contexto, las organizaciones modernas (Daft, 2013). Veamos el concepto, luego el contexto y finalmente el concepto en el contexto.

El concepto: Nizet y Rigaux (2014) sostienen que Goffman fue riguroso e inspirador, más no sistemático; cada libro suponía una nueva variación de sus temas obsesivos. Como el propio Bourdieu (1987) afirmaba de sí mismo, Goffman (1966 y 1961) se resistía a las definiciones cerradas, prefiriendo deslizar su interés de los sistemas naturales cerrados al juego [game]. Entonces, para definir IM, pues, será preciso ponerla antes en relación con el movimiento del pensar de Goffman. Para Goffman (1956) la vida es teatro, y las interacciones con otros, pura dramaturgia de lo cotidiano. Goffman $(1981 ; 1966 ; 1956)$ toma prestado del interaccionismo social de Mead y Blumer el concepto de self. En un extremo, el «yo» $[I]$ es nuestra parte interior, asocial, desorganizada y pulsional; en el otro, el «mí» [me] organiza nuestra vida en sociedad. En medio se ubica el self, esa parte de nuestro ser en continua tensión entre ser y parecer, que se repliega en forma de monólogo o se proyecta al encuentro de otros como conversación o gesto (Charon, 2009). El self no se trata de una posesión estática del sujeto, sino un producto móvil derivado de las interacciones (Goffman, 1956). Por interacción cabe entender el acto por excelencia de la sociología de lo cotidiano: el instante en que dos o más sujetos entran en relación (Fine, 2012; Goffman, 1956 y 1961). La interacción es la unidad mínima empírica: real, observable, constatable y, por encima de todo, aprehensible al estudio sociológico (Charon, 2009; Goffman, 1956; 1986). No obstante, la interacción depende del orden de interacción [interaction order], cosa a tener en cuenta para interpretarla cabal, rigurosamente. El orden de interacción consiste en el sistema de convenciones y normas sociales implícitas que, en una situación concreta, determinan y delimitan cada interacción; el orden de interacción es el texto invisible del drama cotidiano, donde cada actor interpreta su papel, modula su presentación del self.

He aquí el play en su doble acepción: como juego y como obra de teatro (Goffman, 1956; 1966). Toda interacción se basa en esta regulación tácita, en esta economía de la presentación del self (Goffman, 1966) en la que los actores tratan de imponer una presentación de su persona concreta y su propia definición de la situación (Goffman, 1956). Así, todo individuo ejerce una «gestión de la impresión» [impression management] para controlar la situación e influenciar la impresión que otros poseen de él (Goffman, 1959). O en otras palabras, desde la estética cotidiana de Goffman, las personas buscan ser los actores adecuados — la 
presentación idealizada del self — en la obra representada — orden de interacción dado- (Gouldner, 1970). Los individuos actúan imponiendo un retrato determinado sobre sí mismos, para motivar ciertos provechos de esas interacciones (Goffman, 1959).

El contexto: la definición clásica de organización sugiere que esta es un conjunto de actores que se unen deliberadamente y que, a través de relaciones sociales útiles, pretenden conseguir un supraobjetivo específico, el cual estaría más allá de sus posibilidades por separado (Mayntz, 1980). Por ende, las interacciones, comportamientos y hábitos responderán a una lógica que los encauza a procesos productivos tendentes al cometido final de la organización (Daft, 2013). En cierto sentido, no existe nada más social que una organización (Mayntz, 1980), porque ofrece un entorno determinado donde estudiar la socialización compleja (Casey, 2002). De lo que no resulta difícil inferir que la racionalización, el funcionalismo fayoliano y la prioridad de las estructuras sobre los sujetos han sido históricamente los grandes tótems de la teoría organizacional, lo que supone un énfasis en las estructuras en detrimento de los agentes, casi un recurso más (Casey, 2002). En la década de los ochenta, la cultura y el descubrimiento del sujeto abren el estudio de la organización (Morrill, 2008); así, el clásico Cultural Organizations: Fragments of a Theory de Van Maanen y Barley (1985) señala que las organizaciones consisten en sistemas de significados socialmente construidos, donde toda pretensión de homogeneidad estructural resulta una ilusión ante la parcelación y la pluralidad real.

Las interacciones y las transacciones simbólicas entre individuos, grupos y totalidad crean estos mundos sociales (McCarthy, 2013). Autores diversos, de Shulman (2007) a Valenzuela, Reygadas y Cruces (2015), reinciden en las tensiones del self entre la estructura organizacional y el modo de pensar y actuar privado. En órdenes de interacciones organizativos los individuos tenderán a ejemplificar, más que su identidad, los valores y actitudes comúnmente aceptados (Goffman, 1959). Este encaje entre el sujeto y la organización se debe, según el goffmiano Fine (2012), a la posesión de un conocimiento experto de la materia y, más importante, el conocimiento de los procedimientos formales e informales de la socialización. Considerando que existe una carencia explicativa que dé rendida cuenta de la performatividad de los actores, Freidson (1970) añade los escenarios [settings] o entorno de trabajo inmediato, esto es, la materialidad del lugar y de los objetos disponibles.

El concepto en el contexto: crear una imagen social para motivar unos resultados determinados se presta a ser utilizado en los entornos organizacionales. Este concepto goffmiano, que denota la inestable relación de la identidad con su 
contexto social (Zavattaro, 2013), ha atraído la atención de los investigadores organizacionales (Gardner, 1988). En las organizaciones, caracterizadas por la colaboración y, al mismo tiempo, la competitividad (Casey, 2002), la IM, esto es, el proceso por el cual los agentes organizacionales — trabajadores o empleados en latu sensu - buscan influenciar en la imagen que sus compañeros/competidores se forman de ellos, es un elemento crucial (Rosenfeld, Giacalone y Riordan, 1995) y, más interesante, estratégico (Sallot, 2002). Esta exteriorización de una identidad idealizada es la base de la socialización organizacional (Rosenfeld, Giacalone y Riordan, 1995; Bolino y Turnley, 2003; Pollach y Kerbler, 2011), tan embebida que no puede entenderse un currículum (Knouse, 1994), una entrevista de trabajo (Ralston y Kirwood, 1999) o cualquier ocasión para mostrarse competente (Pollach y Kebler, 2011) sin la IM. Cualquier interacción es válida para demostrar méritos, recursos, actitudes, competencia y fiereza de compromiso que beneficien ciertas metas [goals] del agente (Rosenfeld, Giacalone y Riordan, 1995), como, por ejemplo, promociones salariales (Giacalone y Resenfeld, 1991). En consecuencia, además de los significativos trabajos de Turnley, Bolino y Gardner, los estudios de IM en las organizaciones son numerosos y variados: la influencia de las calificaciones del rendimiento de los subalternos según la simpatía con los responsables (Wayne y Liden, 1995), la diferencia de la IM agresiva de los varones (Bolino y Turnley, 2003), el esfuerzo de los empleados en agradar a sus superiores (Drory y Zaidman, 2007), la búsqueda de legitimar acciones (Brown, 1997) o el liderazgo (Gardner y Cleavenger, 1998), la fractura entre objetivos individuales y organizacionales (Mohammadmoradai, Ashrafi y Fakhar, 2014), la lucha por eliminar la imagen indeseada e imponer la idealizada (Turnley y Bolino, 2001) y los efectos sobre las expresiones emocionales (Soran y Balkan, 2013) o, incluso, sobre la difamación (Mohamed y Gardner, 2004). Así, indagar en la IM es indagar en la naturaleza social de la organización (Crane y Crane, 2002): cómo los agentes se influencian entre sí en todas sus interacciones (Bolino y Turnley, 2001).

A pesar de los distintos estudios citados ut supra, hay todavía territorio inexplorado. A pesar de que Goffman se centró en la interacción cara a cara [face-toface], es decir, situación de copresencia, no obviaba la materialidad de estas interacciones (Pinch, 2010; Collins 2004). Nunca llegó a desarrollarla (Pinch, 2010 y 2008), pero con la nebulosa noción de materialidad [materiality] se refirió al propio cuerpo y su ubicación en el espacio (Goffman, 1971) y al sustrato material - texto, voz- de la interacción (Goffman, 1969); asociaba, pues, materialidad de la interacción a la dimensión física: a) el cuerpo de los actores — su posición, su imagen, sus gestos, su voz-, b) el espacio donde tiene lugar la situación y c) 
los objetos o la tecnología comunicacional - el medio- que transporte el mensaje; materialidad que, a la sazón, también determina en parte dicha interacción (Goffman, 1971). La sociología precisa de materialidad (Pinch, 2008), tanto de la tecnología (Pinch, 2010) como de los cuerpos y los lugares; lo social no se da entre voces sin cuerpo, en espacios asépticos y sin que medien los objetos (Le Breton, 2013). A pesar de que la materialidad se abre paso en la sociología, en especial, por los trabajos de Leonardi (2013; y con Barley, 2010 y 2008), donde el objeto tiene un papel latournianamente preponderante, el triple enfoque goffmiano no ha sido integrado todavía (Pinch, 2010). Como en otras intuiciones goffmianas, la ambigüedad dota de libertad al investigador para experimentar (Nizet y Rigaux, 2014), por lo que sobre esta triple noción de materialidad — cuerpo, espacio, objetos - trabajaremos sobre un mundo social concreto: la organización contemporánea. Por ello nos basamos en este hecho no cubierto: las interacciones en entornos organizacionales se llevan a cabo a través de distintas materialidades; los agentes organizacionales asumen distintas dramaturgias a través de sus IM a lo largo del día, y estas poseen una materialidad particular. Centrándonos en la IM, estudiamos situaciones organizacionales con distintas materialidades; a través de los diferenciales que observamos entre las IM, nuestro objetivo es generar el esbozo teórico de la ixtimidad que proporcione nuevos conocimientos de la socialización en organizaciones. Desde la pluralidad de situaciones y su sustrato material entenderemos las tensiones que sufre el self.

\section{Diseño y metodología}

Nuestra investigación se sustenta sobre el dato encarnado en sujetos y que emana de su mundo simbólico; a este se accede con metodologías cualitativo-inductivas (Yin, 2011). Este enfoque, que Taylor y Bogdan (1987) denominan fenomenológico, los objetos, hechos y fenómenos existen por mediación de significados socialmente construidos. Ahí radica el quid: desmadejar los símbolos entrelazados que están anclados a cuerpos, espacios y situaciones (Yin, 2011). Así distinguimos lo casual de lo causal. A tales efectos el diseño metodológico fue la Grounded Theory, que comunica la captación de datos empíricos - realidadcon la construcción de codificaciones que, a medida que madura la captación y maduran las versiones, da lugar a categorías y estas, convenientemente agrupadas, a asertos teóricos (Charmaz, 2006; Glaser y Strauss, 1967). No entramos con teoría predeterminada, construimos rigurosamente teoría a partir de la realidad fenoménica (Clarke, 2005). Aplicamos este diseño a una muestra empírica constituida por unidades de gestión transversal — departamentos, direcciones - en siete distintas organizaciones; para Mintzberg (1990), estas corresponden a las 
más transversales, transectoriales y de funciones de una organización a otra, comparativamente hablando, más análogas. Acotada la muestra a una praxis laboral, apegamos nuestra investigación a prácticas concretas y no a discursos corporativos (Freidson, 1970). La anonimidad en etnografía organizacional es fundamental (Morente 2017; Ladner, 2014); tras firmar los consentimientos informados, omitimos mención alguna de las actividades y eliminamos cualquier atributo de las organizaciones y los participantes. La muestra comprendía un caso de estudio central (CEC) y seis casos de estudio satélite (CES); la diferencia estriba en que en el CEC empleamos, adicionalmente, la etnografía intensiva. La anonimidad en etnografía organizacional es fundamental (Morente 2017; Ladner, 2014); tras firmar los consentimientos informados, omitimos mención alguna de las actividades y eliminamos cualquier atributo de las organizaciones y los participantes. La muestra comprendía un caso de estudio central (CEC) y seis casos de estudio satélite (CES); la diferencia estriba en que en el CEC empleamos, adicionalmente, la etnografía intensiva. En cuanto fuente de datos, cada caso era una petite generalization (Stake, 1995); no obstante, con la conexión del CEC con los seis CES pretendíamos romper los particularismos y potenciar lo que Einsenhardt y Graebner (2007) denominan la «lógica de la replicación» [logic of replication], esto es, que nuestras generalización e inferencias no solo explicaran los propios casos sino que fueran capaces de explicar casos similares. Así las siete unidades de gestión transversal pertenecían a tamaños, grados de madurez y sectores de actividad diferentes: utility multinacional del agua, pyme del sector metalúrgico, utility multinacional de la energía, universidad privada, fundación sin ánimo de lucro, banca internacional y consultoría multinacional. Identificaremos a los participantes como CEC o CES, en función de su adscripción a una organización u otra.

Sobre esta muestra desplegamos tres fases trianguladas:

Fase I. 12/2015 - 06/2016. Centrada en captar datos de 1) bibliografía especializada sobre la gestión transversal, teoría organizacional, sociología interaccionista, 2) documentación corporativa - webs, annual reports, etc. - y 3) etnografía aplicada en CEC. El etnógrafo es un «extraño profesional» (Agar, 1996); observa e indaga en la cotidianeidad de los participantes para entender desde dentro - emic - intenciones, motivos, actitudes y creencias (Hammersley y Atkinson, 2007), y que, con tiempo, hará aflorar patrones (Ladner, 2014). La etnografía fue de más de 1.700 horas en oficina, lugar de la actividad principal del CEC, y dejando a un lado encuentros fuera del horario laboral (afterworks) y actividad en redes sociales. Para no contaminar el dato, hicimos investigación discreta [unobstrusive research] (Webb et alii, 1966); buscamos información cor- 
porativa sin pasar por ellos y tratábamos de volver invisible nuestra etnografía. Como el dato etnográfico no se disocia de su contexto situacional (Collins, 2004; Agar, 1996), nos centramos en situaciones con o sin mediación de la tecnología - teléfono, Skype, Whatsapp, etc.—, por lo que durante la jornada laboral se etnografiaron reuniones formales, comités, presentaciones, desayunos, comidas y charlas informales.

Fase II. 06/16 - 11/16. Alcanzada en junio la saturación teórica (Glaser y Strauss, 1967), y enriqueciendo conceptos con observaciones empíricas (Yin, 2011), abrimos la etnografía dirigida a interactuar con las versiones del coding. Se realizaron 28 versiones hasta conseguir categorías robustas; un proceso continuo de construcción y destrucción (Strauss y Corbin, 1998), donde afinar la etnografía clarificaba las explicaciones (Agar, 1996). En noviembre, fortaleciendo el coding, realizamos entrevistas cualitativas semiestructuradas a los participantes CEC y CES, con una duración media de hora y tres cuartos. A través de entrevistas se accedió a los materiales simbólicos que construyen los mundos íntimos (Weiss, 1995).

Fase III. 11/16 - 02/17. Los últimos meses se dedicaron a analizar con las cinco fases de Yin (2011). Con el coding casi definitivo emprendimos la estructuración final, buscando conexiones, patrones, conclusiones y nuevos conceptos (Charmaz, 2006). Al concluir, podíamos describir y explicar la realidad fenoménica estudiada.

\section{Resultados y discusión}

Cotidiana, constantemente, entramos en y salimos de distintos órdenes de interacción; y nuestra persona — máscara pública — se amolda a las situaciones (Goffman, 1971). Para profundizar en la IM, en nuestra investigación distinguimos las situaciones tal como sigue (fig.1).

Figura 1. Situaciones estudiadas

\begin{tabular}{|c|c|c|c|}
\hline \multicolumn{3}{|c|}{ Interacciones en oficina } \\
\hline \multicolumn{2}{|c|}{$\begin{array}{c}\text { Mediatizadas } \\
\text { Interacción mediatizada por la tecnología }\end{array}$} & \multicolumn{2}{c|}{$\begin{array}{c}\text { En copresencia } \\
\text { Interacción cara a cara }\end{array}$} \\
\hline $\begin{array}{c}\text { E-mail, } \\
\text { chat, Whatsapp }\end{array}$ & $\begin{array}{c}\text { Teléfono y } \\
\text { videoconferencia }\end{array}$ & Reuniones & Desayunos \\
\hline $\begin{array}{c}\text { Ausencia de cuerpo y } \\
\text { de voz }\end{array}$ & $\begin{array}{c}\text { Ausencia de cuerpo, no } \\
\text { de voz }\end{array}$ & $\begin{array}{c}\text { Espacios/tiempos } \\
\text { organizacionales }\end{array}$ & $\begin{array}{c}\text { Espacios/tiempos } \\
\text { residuales }\end{array}$ \\
\hline
\end{tabular}

Fuente: elaboración propia. 
La distinción estriba en la materialidad de los encuentros y no en la formalidad, una variable, como así observamos y algunos autores sugieren (Wang y Ahmed, 2002), de inmensa plasticidad social. Para mantener la triple materialidad goffmiana - cuerpo, espacio, objetos-, nuestras dos grandes categorías son situaciones mediatizadas y situaciones con copresencia. Por mediatizada nos referimos a las interacciones habilitadas por la tecnología, tanto por el poder tecnológico para crear nuevas situaciones como por la forma que tienen los agentes de utilizarlas (Richley, Ravishankar y Coupland, 2016); por copresencia, cara a cara (Goffman, 1971, 1966). De este modo, exponemos de nuestra muestra la descripción densa [tbick description] à la Geertz (1973) de las situaciones.

\section{E-mails, chats, Whatsapp}

Por su presencia masiva en oficina, el e-mail es una forma de interacción por derecho propio y una de las principales comunicaciones mediatizadas por ordenadores (Waldvogel, 2007). Los participantes no eran ajenos: [en la cafetería] «Te lo juro. A veces tengo la sensación de que solo envío e-mails» (risas) (CEC, 19/07/16); o [en las mesas de trabajo] «Fíjate (señala la pantalla) todos estos correos. Es una locura» (CEC, 7/III/16). En cuestiones de IM formal, el e-mail presentaba varias ventajas a ojos de los participantes y varias inferencias para el investigador. La más evidente era la instantaneidad: «En cuanto envías el correo, te olvidas. El trabajo está en su tejado» (CES, 22/XI/16). A expensas del cuerpo y la voz, los e-mails adolecen de profundidad al dirigirse directamente al propósito comunicacional (Waldvogel, 2007). Esta incorporeidad facilita que los participantes actúen sin circunloquios — «Por correo siempre vas a saco» (CEC, $31 / \mathrm{X} / 16)$ - , puesto que la exposición física es nula, esto es, segura — «Escribir el e-mail siempre para estos temas es más sencillo que decirlo a la cara, ¿no?» (CES, 5/XII/16) - . El e-mail es diacrónico, no busca el diálogo ni la negociación; segmenta temporalmente la interacción — «Fíjate lo que ha puesto. ¿ Me espero y le contesto esta tarde?» (CEC, 7/IX/2016) —. Dada su instantaneidad diacrónica el e-mail agilizaba la acción, de ahí su tránsito constante. En e-mail, la IM era formal, pues, en caso de conflicto, podía hacer las veces de prueba telemática: «Por si las moscas, no llames. Mejor enviar un correo para que quede constancia. Nunca se sabe» (CEC, 18/II/16). Cual libro de contabilidad, permitía trazar las interacciones, datando los avances del trabajo e indicando las atribuciones. En esta misma línea, la función de «poner en copia» confería al e-mail la legitimidad organizativa necesaria para asignar acciones: «No hay nada como poner a un responsable en copia. Pidas lo que pidas está... cómo te diría... validado» (CES, 29/XII/16). Con su sola inclusión — que simboliza los valores estructurales-, 
un correo legitimaba acción o coaccionaba. Observamos que a mayor cantidad de remitentes tanto mayor el grado de formalismo, de despersonalización - esto es, renunciar a su persona en favor de comportarse acorde a su rol (Powers, 1981) _. En cuanto elemento transaccional que registra interacciones (Waldvogel, 2007), también era el momento de mostrar la adhesión a las normas tácitas de la organización, e incluso, la sobreadhesión, con e-mails fuera del horario laboral o en fines de semana. Crozier y Friedberg (1977) predicen que en (tele)presencia de actores con un grado variable de confianza las personas actuamos enfatizando el compromiso organizacional. En el juego organizacional los e-mails proponen situaciones en las que los actores rehúyen de una singularización negativa en pos de una adhesión ostentativa a la organización, porque, recalca Zerubavel (2006), conocer las normas demuestra la pertenencia a la comunidad. Nos apercibimos de que los e-mails solían ser escrupulosamente formales y, por ende, la exposición del self, mínima. Se cuidaba la expresión, los giros y las opiniones personales se ceñían al orden oficial. Esta situación venía motivada no solo por la exposición a otros, sino por un factor más: el almacenaje de la interacción. La materialidad del e-mail no precisa de cuerpos — elimina gestos y voz — ni de espacios — abole el tiempo compartido-, pero registra la interacción; que la palabra escrita posea una materialidad imperecedera, independiente y en manos de la organización significa que puede guardarse, malinterpretarse y difundirse más allá del propósito inicial. Todo e-mail atenta contra la IM, porque nuestra imagen se independiza. Por este motivo, el chat interno tenía un uso extremadamente residual y apenas se utilizaba. Goffman (1966) señala que en situaciones en que nuestras palabras pueden almacenarse habrá inhibición: [por chat] «Buf, paso por aquí. Mejor por whatsapp» (CEC, 5/VIII/16); y [por correo] «¿Lo hablamos con un café?» (CEC, 3/XI/17). Con un orden de interacción alternativo al organizacional, y procurando que no fueran corpus delicti, los e-mails informales solían ser escuetos, elípticos, alusivos — «Qué show!»-y emplazaban a otra situación con una materialidad más efímera.

Por el contrario, Whatsapp suponía una cierta relajación en la IM. Wägar (2012) sostiene que lo mundano, aunque no evidente, es la columna vertebral de las organizaciones; en el Whatsapp tenía lugar esa mundanidad mediatizada. Se compartían memes, vídeos virales, noticias de la actividad departamental y comentarios irónicos de su realidad laboral. Con Fine (2012) vinculamos ironía a alejamiento de la ortodoxia y la interposición del humor: un desvío. Los whats contenían motes, emoticonos, dobles sentidos, cotilleos y comentarios desenfadados sobre el trabajo, más no sobre la vida personal. Hacemos dos lecturas. Por una parte, el Whatsapp no se percibía como una herramienta organizacional, por 
lo que, al no almacenarse en sus servers, las conversaciones se desnormativizaban. He aquí un límite de lo que Marx (2017) denomina «organización omnisciente»; más abiertos que en el chat, los participantes se sienten más allá de la esfera organizacional. Por otra, no acaban de sustraerse mentalmente de esta. En el trabajo, el mundo se reduce a lo laboral, y este es el sustrato interaccional (Freidson, 1970); así, siempre subyace el control de la IM: cercanía, desinhibición, pero rechazo a abrirse. Por otra, no acaban de sustraerse mentalmente de esta. En el trabajo, el mundo se reduce a lo laboral, y este es el sustrato interaccional (Freidson, 1970); así, siempre subyace el control de la IM: cercanía, desinhibición, pero rechazo a abrirse. El contexto común propicia la mecanización social (Goffman, 1961), y en este caso el trabajo supone el material contextual sobre el que se reelabora la interacción. La intimidad es territorio vedado.

\section{Teléfono y videoconferencia}

En comparación con los tres anteriores medios, el teléfono permite un paralenguaje que añade emotividad a la interacción: la voz (Bailenson et al, 2004). La voz expresa, emociona, suscita el recuerdo del cuerpo ausente (Collins, 2004). Las llamadas telefónicas se sucedían sin descanso; los participantes utilizaban el teléfono para 1) eliminar los momentos muertos de espera de respuesta — «Levantas el teléfono y lo solucionas enseguida» (CES, 29/XII/16) —, 2) habilitar una conversación oral no registrada — «Esto por e-mail mejor que no..»» (CES, 7/XII/2016)—, 3) ahorrar tiempo al eludir la escritura — «Hola... te llamaba para no soltarte la parrafada» (CES, 6/XII/2016)—, y, gracias a la expresividad de la voz, 4) conseguir una inferencia interpretativa más pulcra o incrementar el control de la propia expresividad: «Normalmente llamo porque así pillas más matices y te explicas mejor. Por correo se pierde mucho» (CES, 18/VII/2016); y «¿Has visto lo que me ha puesto [en el e-mail]? ¡Lo llamo a ver qué quiere!» (CEC, 14/IX/2016). La voz directa y transparente aporta inferencias interpretativas — «matices» al decir de los participantes_-, información que ayuda a controlar su IM (Bailenson et al., 2004; Goffman, 1966). En la interacción telefónica se comparte la temporalidad vivida y, en cierto sentido, es íntima, pues facilita la negociación en tiempo real (Fortunati, 2005), porque al «llamar» se busca el cuerpo sugerido por la voz (Laberge, 2016): «[con enfado] Estoy seguro. Está en su mesa y no me lo pilla» (CES, 25/XI/2016). Al contrario que el e-mail, la llamada telefónica es instantánea y sincrónica. En las situaciones en ausencia de cuerpo pero con voz, se añade la videoconferencia; la irrupción de Skype y Webex supone una nueva herramienta en los puestos de trabajo cada vez más deslocalizados espacialmente (Denstadli et al., 2013). A la voz se añade la imagen. Etno- 
gráficamente vimos que la imagen compartida no es tanto la suya cuanto la de la documentación. Reticentes a compartir su imagen, los participantes hacían un uso utilitarioः «Con la video[conferencia] compartes el PowerPoint y es más fácil entenderse porque ves lo mismo» (CEC, 29/VI/2016); «Siempre tiene su qué ver al otro, porque no tienes que imaginártelo pero casi siempre saludas y pones el documento» (CES, 29/XII/2016). Se prefiere ver el documento, que favorece la conversación útil; la imagen propia o del otro halla dos escollos tecnológicos que merman su utilidad social para la IM: 1) la cam — «No aporta mucho. Ves al otro encajonado»(CES, 29/XI/2016) - y 2) el lag — «La imagen y la voz no van juntas y cuesta entender» (CEC, 9/I/2016) - . Aspectos que revelan la artificialidad de la expresión corporal (Le Breton, 2013); un cuerpo que, a efectos de la IM, está mudo. La opinión y la práctica de los participantes encajaba; la videoconferencia era «[... $]$ para reuniones de segunda división [... Cuando la cosa es más seria, hace falta una reunión» (CES, 22/XI/2016). A pesar de la temporalidad compartida de estos dos medios, cuando es preciso negociar, se acude a la reunión: a la aparición plena del cuerpo.

\section{Reuniones}

La reunión es el encuentro organizacional por antonomasia, pues engrana el proceso de socialización al mostrarse, confirmarse y compartirse los valores y las metas oficiales (Boden, 1994), al tiempo que supone el instante de negociación y decisión (Daft, 2013). Los participantes, inconscientemente, perciben esta importancia, tanto de valor simbólico como de su utilidad: «Hay que preparar bien la reunión. No subimos eso para hacer el ridículo» (CEC, 8/III/2016); «Uno se puede equivocar, pero nunca en una reunión. Están los ojos puestos en ti» (CES, 22/XI/2016). También intuyen, por la práctica cotidiana, el poder de la presencia del cuerpoः «El correo se queda corto. Mejor la reunión. Así los veo, me ponen cara. Por Webex los ves, pero no es lo mismo que tenerlos delante» (CEC, 8/V/2016); "Si hacen la reunión y tú estás por teléfono, notas que te pierdes algo» (CES, 29/XI/2016); y «Si hay que decidir, por mucho que hable por Webex, no harán caso. Tengo que estar ahí» (CES, 26/XI/2016). El encuentro con cuerpos despierta emotividad y recelo a partes iguales (Le Breton, 2013; Sacks, 2006), y en las reuniones a las que asistimos detectamos tres factores corporales: 1) encuentro directo - vestimenta, proximidad, visibilidad, voz sin mediación, lenguaje corporal..., son signos interpretables en los otros y controlables en nosotros - ; 2) rostro — cuando dos miradas se cruzan se reconoce el estatus y la valía personal y laboral-; y 3) improductividad — los tiempos muertos al abrir un archivo o subir el aire acondicionado muestran la fragilidad de la situación 
y motivan silencios elocuentes que es preciso llenar-. La reunión, pues, supone copresencia, el cara a cara con el otro (Goffman, 1966): proximidad, profundidad interaccional, control de la IM y capacidad de mediación. Las reuniones solían realizarse en el primer espacio oldenburgiano (1989), el espacio laboral - despacho, salas de reuniones o mesas de trabajo que simbolizan los espacios de poder-; en términos goffmianos, la representación oficial tenía lugar en el «escenario» [front stage]. La reunión, pues, supone copresencia, el cara a cara con el otro (Goffman, 1966): proximidad, profundidad interaccional, control de la IM y capacidad de mediación. Las reuniones solían realizarse en el primer espacio oldenburgiano (1989), el espacio laboral — despacho, salas de reuniones o mesas de trabajo que simbolizan los espacios de poder-; en términos goffmianos, la representación oficial tenía lugar en el «escenario» [front stage]: «Eso no lo va a decir en la sala [de reuniones] y con más gente delante» (CES, 29/XI/2016); «Es el momento [la reunión] de ser competente»(CES, 5/XII/2016). Además de discurrir en espacios «oficiales», estas eran extremadamente formales, muestra de ello son las excesivas «invitaciones» [invitations] (Sacks, 2006); las charlas sin intimidad se iniciaban con fórmulas verbales vacías de implicación interaccional: preguntas sobre los hijos, la climatología o el tráfico, o comentarios acerca del volumen de trabajo. Estas tenían por objetivo sintonizar a los actores en la misma longitud de onda, es decir, construir los estrechos márgenes del orden de interacción donde la IM se ajustará a la doxa y los modos organizacionales. Los participantes debían mostrar que su actitud y sus formas encajan con el mundo social de la organización; no obstante, al mismo tiempo, sabían que no podían conformarse con presentar su self integrado en la organización: «Es el momento de lucirse. Que se note uno. Destacar» (CES, 22/XI/2016); «No hay muchos momentos de visibilidad. La reunión con los jefes es una oportunidad» (CEC, 14/VI/2016). En relación con la organización, la IM debía encajar en el conjunto normativo tácito organizacional y, al mismo tiempo, singularizar al sujeto del resto de empleados. Los actores en las reuniones, y más en presencia de jerarcas, proyectan imágenes idealizadas del self: la del empleado sin tacha (Zavattaro, 2013). Esta idealización dramatúrgica observada conjuga la integración despersonalizada — mezclarse con la masa organizacional — con la singularización individuarse de esta-. El único margen de singularización que observamos no fue la réplica renovadora, sino la exageración estructural del orden prestablecido. Los individuos organizacionales tienen sus propias metas que no siempre coinciden con las de la organización (Mayntz, 1980), y en las reuniones, a través de la representación hiperbólica de los valores organizacionales, los agentes muestran la existencia de aspiraciones personales. Debajo del aparente orden homogéneo, bulle una multitud de individuos. 


\section{Desayunos}

La organización informal [informal organization] comprende la subvida [underlife], los lazos débiles [weak ties] y la normatividad alternativa (Casey, 2002). Toda centralización organizacional tiene su reverso informal (Crozier y Friedberg, 1997), plagado de microscópicas excepciones (Van Maanen y Barley, 1985), banales, no productiva pero socialmente significantes (Javeau, 1991). En nuestra muestra, estas excepciones eran los desayunos. Los desayunos se realizaban en la cafetería de la sede; un segundo espacio oldenburgiano, pero con atributos del tercero: terreno neutro en el que nadie asume el rol de anfitrión, inclusivo y donde la conversación es la actividad principal. Este espacio despertaba un sentimiento de recogimiento, protección y distensión del self, hecho que se dejaba sentir en la postergación de ciertas charlas: «Oye, ¿subimos y comentamos [tema sensible] con un café?» (CEC, 27/V/2016); «Durante el café hablas más libremente. Si hay buen rollo, hay más confi[anza]» (CEC, 8/III/2016). Así, comprobamos en campo que este espacio, en los márgenes de la actividad laboral, propiciaba un deslinde interaccional en la IM. Otro factor es la selección de los interlocutores; en un principio, los participantes eran libres de elegir con quién desayunar — «Deja el PowerPoint para después, y vamos a subir [a la cafetería]» (CEC 26/IX/2016) - Al promediar las 9:30, los participantes buscaban interlocutores o sus grupos afines; solían reunirse por estratos jerárquicos $y$, raramente, los técnicos desayunaban con responsables o directivos o con quienes se carecía de confianza — «Vamos rápido a desayunar que [X] se nos acopla» (4/V/2016)—; no había lugar para la representación hiperbólica de las reuniones. Esto nos ponía sobre la pista de que existía cierta intimidad en este tipo de interacciones y que el cometido solía centrarse en el «comentario social» de asuntos organizacionales, tales como contratiempos/problemas — «pollos»o «brownies»—, cambios jerárquicos —uúltimos movimientos»—, resolución de problemas laborales — «echar un cable»-y vidas sentimentales dentro de la organización - «cotis»-. Las confidencias marcan un nuevo grado de confianza (Fine, 2012). Sin embargo, nunca se debatían posturas ideológicas o compartían temas personales; por el contrario, a base de sondear a los otros vertiendo opiniones inanes, se buscaban el consenso y el reconocimiento. Nuevamente, como en Whatsapp, a pesar de dejar atrás la máscara del rol, los participantes solían volcar sus morigeradas opiniones subjetivas sobre temáticas laborales. Por ende, la concentración emocional en los desayunos distaba de la observada en reuniones o e-mails: «A Ahora no te hagas el longuis y cuenta! $\underset{i}{ } \mathrm{Va}$, cuenta eso tan importante que tenías que explicar! No vale ser polite» (CEC, 8/II/2016); «Eso no lo decías "así" hace diez minutos en la reunión, ¡eh!» (CEC, 28/X/2016). La oralidad, como 
cuarto factor, favorecía también otro tipo de IM: informal, coloquial y, en ocasiones, inocentemente subversiva — «Esto te lo cuento, pero que no salga de esta mesa» (CEC, 18/VII/2016). Cuando los mensajes eran efímeros, sin registro ni memoria, cuando estos quedaban depositados en la memoria de otros y no eran mediatizados, la palabra se desligaba de la jerga y de la manipulación de la apariencia, y parecían orientarse hacia un tono confesional, pero con salvedades. $\mathrm{Al}$ observar la implicación (Goffman, 1966), la expresión de un propósito personal, concluimos que se prioriza la baja exposición, que contribuye a crear un orden de interacción seguro y cauto, nunca comprometedor para la IM. A tenor de lo expuesto, los desayunos padecen de las pequeñas inhibiciones (Goffman, 1966): pese a las valoraciones más abiertas en lo tocante a ciertos aspectos laborales, las conversaciones nunca llegan a ser íntimas en su sentido pleno y, por ende, la exposición del self está sesgada a cierto formalismo dentro de la informalidad; persiste el terror a la malinterpretación — «Hay temas que tienes que ir con cuidado. Siempre puedes herir sensibilidades si se interpreta mal» (CES, 7/XII/2016)— y no se olvidan del gran marco en el que se insieren sus interacciones — «Que sí, hablas más suelto..., pero, aunque te eches unas risas, no olvidas que estás en el curro» (CES, 22/XI/2016)-.

\section{Conclusiones}

Sintetizamos la thick description. 1) Interacciones mediatizadas. a) El e-mail (y el chat) es incorpóreo, instantáneo, poco profundo y diacrónico; al almacenar las interacciones como datos que desligan la IM de sus contextos originales, los agentes muestran una exposición nula de su self, muy formal y se despersonalizan por mor de encajar con la doxa organizacional. Desde su registro informal, las interacciones en Whatsapp ahondan en la fuerza de la apariencia y la cautela: solo versan del trabajo, único asidero interaccional. b) El teléfono, a su vez, añade la expresividad emotiva de la voz — recuerdo de un cuerpo ausente y fuente de nuevos matices interpretativos - y la sincronía, que abre las puertas a la negociación. La videoconferencia, por el contrario, se muestra como un medio artificial a causa del lag y la cam, que enmudecen el cuerpo. 2) Interacciones en copresencia. a) Las reuniones, principal herramienta de negociación, se llevan a cabo en espacios oficiales y ante extraños, por lo que resultan formales y tensas; la IM, expuesta, se debate entre encajar con el conjunto de valores organizacionales - despersonalización-y la singularización del sujeto a través de la representación hiperbólica que exagera la adhesión al status quo. b) Los desayunos resultan, en apariencia, informales; no obstante, a la luz de las pequeñas inhibiciones y la temática eminentemente laboral de las charlas, no se puede hablar de una IM informal, puesto 
que en todas las interacciones, hasta en las más nimias, la fantasmagoría organizacional se desliza en ellas.

De lo que se desprende que, para adecuar su IM, los participantes captan y procesan datos de la materialidad constantemente para actuar consecuentemente sobre el control y la influencia de las situaciones; en palabras de Sacks (2006), hacen inferencias [inferences] con las que construyen su conocimiento del entorno y valoran cuál es la mejor imagen a proyectar en los otros en un entorno social tan singularmente dado a la actuación y a la interacción estratégica. La sobreexposición conlleva peligros —interpretaciones complejas, improvisación - pero también beneficios - capacidad de singularización y de control de la situación-. Ladner (2014) advierte de que la etnografía organizacional debe asumir la existencia de un grado máximo de veracidad en las expresiones del self. Cualquier agente posee sus objetivos e intereses particulares, su propia estrategia (Crozier y Friedberg, 1997). En consecuencia, el self organizacional se encuentra inmerso en este debate ¿cómo adecuar la IM a los imperativos de la despersonalización y a la singularización?

Proponemos un concepto sociológico de nuevo cuño. En las organizaciones modernas los actores deben jugar al «doble juego», pues deben demostrar en todo momento con sus IM que conocen el juego — el game, según Goffman (1966)—, que encajan, que se despersonalizan, que reconocen un orden social determinado y que saben desenvolverse en él según una normatividad tácita, pero, al mismo tiempo, puesto que poseen metas individuales tales como prosperar económicamente, conseguir estatus o ascender en la jerarquía piramidal, se ven impelidos a singularizarse respecto al grueso de la plantilla gracias al «juego» - el jeu, según Crozier y Friedberg (1977) —, el conjunto de acciones sobre la IM con base en los objetivos privados. En este doble juego, los actores mostrarán adhesión a la normatividad organizacional, pero si desean singularizarse del resto de compañeros/competidores es preciso que redoblen esfuerzos. Si la vida es una representación - el play goffmiano (1956) —, en las organizaciones el game y el jeu conviven, se solapan, se necesitan y nunca se anulan; casi cabría afirmar que las organizaciones parecen asegurar su pervivencia a través de agentes que se singularizan mediante la representación hiperbólica, la cual contribuye a legitimar su régimen de realidad interno. Por esta razón incluso los objetivos personales más alejados contribuyen al supraobjetivo organizacional.

Atendiendo a la paradoja actoral del doble juego en las organizaciones, definimos el concepto de ixtimidad. Traemos a colación el neologismo de Michel Tournier (2004) para describir la relación que no es íntima y personal, pero tampoco externa o epidérmica; relación, pues, entre pares que hablan íntimamente, 
aunque nunca de sus vidas privadas — inquietudes, deseos, jeu-, sino de su realidad laboral circundante. Relación fronteriza entre lo personal e informal con lo laboral y formal, definimos la relación íxtima como aquella por la cual los agentes expresan con cierta libertad sus opiniones pero en relación a un material vivencial común y externo, en este caso el trabajo. Esto permite que la IM asuma formas personales, informales y no estrictamente supeditadas a la normatividad organizacional, pero sin ofrecer argumentos a desenmascararse. Los actores interaccionan íxtimamente porque de este modo muestran su adhesión a la compañía y cabe la posibilidad de singularizarse del resto; al apoyarse la interacción en temas laborales, y nunca personales, los actores pueden volcar sus opiniones personales sin exponerse a riesgos innecesarios - la vergüenza, la envidia, el descrédito-, los cuales pueden llevar aparejadas consecuencias que vayan en detrimento del futuro laboral. La ixtimidad conjuga las actuaciones presentes con los escenarios futuros; una palabra destemplada puede acarrear problemas venideros, una conversación llevada con astucia puede suponer un posible aumento de sueldo. Así, la ixtimidad contribuye a la ambigüedad observada a microescala interaccional de la IM, ambigüedad que provoca interacciones más complejas que las que predice la teoría organizacional clásica, mecánicas y dirigidas a unos fines concretos; el self se desvela ante otros en igual medida que se oculta. Por seguir con las metáforas lúdicas, nunca muestran del todo sus cartas. Con la ixtimidad los agentes ensanchan las maniobras de gestión de su impresión, hasta el punto incluso de la semitransparencia: dejar intuir sus objetivos íntimos para hallar compañerismo o fraguar una microalianza que les permita alcanzarlos sin correr riesgos innecesarios. Es con la ixtimidad como se ocultan las estrategias individuales o se semidesvelan, siempre en jugadas estratégicas en las que la veracidad no es el factor principal, sino el efecto social de la palabra. En último término, la ixtimidad, esa frontera difusa, es una muestra de que el sujeto singularizado tiene cabida en las organizaciones, pero este ha de saber jugar con las apariencias, las materialidades y con los elementos culturales de la organización, y orquestarlos en su favor. Ha de saber jugar.

Investigar la IM desde su materialidad múltiple ayuda a profundizar en la socialización cotidiana en el seno de las organizaciones. Los agentes organizacionales luchan diariamente con la imagen que proyectan; rechazan verse atrapados en imágenes perjudiciales que den al traste con sus ambiciones. Con la ixtimi$\mathrm{dad}$, que permite la existencia del doble juego, se pone en evidencia la pluralidad contextual, la heterogeneidad de las estrategias y la maleabilidad de lo que antaño juzgábamos estático, la identidad; un deslinde múltiple hacia un concepto de identidad que, tal y como recordaba el genio de Derek Parfit (1986), siempre es en relación con. 


\section{Bibliografía}

Agar, Michael (1996). The professional stranger. An informal introduction to ethnography. New York: Academic Press.

Alter, Norbert (2013). L'innovation ordinaire. Paris: PUF.

Bailenson, John N.; Beall, Alice C.; Loomis, John; Blascovich, Jeremy y Turk, Michael (2004). "Transformed social interaction: decoupling representation from behavior and form in collaborative virtual environments». Presence, 13(4): 428-441.

Bam, Nancy K. (2015). Personal connection in the digital age. Malden: Polity.

Boden, Deirdre (1994). The business of talk. Cambridge: Polity Press.

Bolino, Mark C. y Turnley, William H. (2003). «More than one way to make an impression: exploring profiles of impression management». Journal of Management, 29(2): 141-160.

Bouncken, Ricarda; Brem, Alexander y Kraus, Sascha (2016). «Multicultural teams as sources for creativity and innovation. The role of cultural diversity on team performance». International Journal of Innovation Management, 20(1): 25-78.

Bourdieu, Pierre (1987). Choses dites. Paris: Éditions de Minuit.

Brown, Andrew (1997). «Narcissism, identity, and legitimacy». Academic of Management Review, 22(3): 643-686.

Casey, Catherine (2002). Critical analysis of organizations. Theory, practice, revitalization. London: Sage.

Charmaz, Keith (2006). Constructing grounded theory. A practical guide through qualitative analysis. London: Sage.

Charon, Joel M. (2009). Symbolic interactionism. An introduction, an interpretation, an integration. New Jersey: Prentice Hall.

Clarke, Adele (2005). Situational analysis. Grounded theory after postmodern turn. Thousand Oaks, USA: Sage.

Collins, Randall (2004). Interaction ritual chains. Princeton: Princeton University Press.

Crane, Edward y Crane, Frank G. (2002). "Usage and effectiveness of impression management strategies in organizational settings». Journal of Group Psychotherapy, Psychodrama and Sociometry, 25:25-34.

Crozier, Michel y Friedberg, Edhard (1977). Lacteur et le système. Les contraintes de l'action collective. Paris: Éditions du Seuil.

Daft, Richard L. (2013). Organization theory E design. Mason: South-WesternCengage Learning. 
Denstadli, John M;; Grupsrud, Manfred; Hjorthol, Richard y Julsrud, Thomas E. (2013). «Videoconferencing and business travel: do new technologies produce new interaction patterns?». Transportation Research, Part C (29): 1-13.

Drory, Alon y Zaidman, Nicholas (2007). «Impression management behavior: effects of the organizational system». Journal of Managerial Psychology, 22: 290-308.

Eisenhardt, Kathleen M. y Graebner, Melissa (2007). «Theory building from cases: opportunities and challenges». Academy of Management Journal, 50:20-24.

Fine, Gary A. (2012). Tiny publics. A theory of group action and culture. New York: Rusell Sage Foundation.

Fortunati, Leopoldina (2005). «Mobile telephone and the presentation of self». En Rich Ling y Per E. Pedersen (eds.) (2005). Mobile communication: re-negotiation of the social sphere. London: Springer.

Freidson, Eliot (1970). Profession of medicine. A study of the sociology of applied knowledge. New York: Dodd, Mead \& Company.

Gaglio, Gérald (2011). Sociologie de l'innovation. Paris: PUF.

Gardner, William L. (1988). «Impression management in organizations». Journal of Management, 14(2)ः321-338.

Gardner, Wiliam L. y Cleavenger, Daniel (1998). «Impression management strategies associated with transformation leadership at the world class level». Management Communication Quarterly, 12(1):3-41.

Geertz, Clifford (1973). The interpretation of cultures. New York: Basic Books.

Giacalone, Robert A. y Rosenfield, Paul (eds.) (1991). Impression management in the organization. Hove: Psychology Press.

Glaser, Barney G. y Anselm L. Strauss (1967). The discovery of grounded theory. Strategies for qualitative research. New York: Aldine.

Goffman, Erving (1956). The presentation of self in everyday life. Edinburgh: University of Edinburgh.

Goffman, Erving (1961). Encounters. Two studies in the sociology of interaction. Indianapolis: Bobs-Merrill.

Goffman, Erving (1966). Behavior in public places. Notes on the social organization of gatherings. New York: Free Press.

Goffman, Erving (1971). Relations in public. Microstudies of the public order. New York: Basic Books.

Goffman, Erving (1981). Forms of Talk. Philadelphia: University of Pennsylvania. 
Goffman, Erving (1983). «The interaction order». American Sociological Review, $48(1): 1-17$.

Goffman, Erving. (1986). Frame analysis. An essay on the organization of experience. Lebanon: University Press of New England.

Gouldner, Alvin (1970). The coming crisis of western sociology. London: Heinemann.

Hammersley, Martyn y Atrinson, Paul (2007). Ethnography. Principles in practice. New York: Routledge.

Hoholm, Thomas y Araujo, Luis (2011). «Studying innovation processes in real-time. The promise and challenges of ethnography». Industrial Marketing Management, 40:933-939.

Javeau, Claude (1991). Au degré zero de la vie quotidienne: les symboles de la banalization. La Societé au Jour le Jour. Paris: PUF.

Jensen, Tommy y Sandröm, Johan (2015). «Normal deviants and Erving Goffman: Extending the literature on organizational stigma». Nordic Journal of Working Life Studies, 5(4): 125-142.

KNouse, Stephen B. (1994). «Impression of the resumé: the effects of applicants education, experience, and impression management». Journal of Business $\mathcal{E}$ Psychology, 9(1): 33-45.

Laberge, Yves (2016). «La theórie d'Erving Goffman sur l'interaction face-à-face pour comprendre le téléphone mobile et les SMS». Recherches Sociologiques et Anthropologiques, 47(1): 205-211.

Ladner, Sam (2014). Practical ethnography. A guide to doing ethnography in the private sector. Walnut Creek: Left Coast Press.

Le Breton, David (2013). Anthropologie du corps et modernité. Paris: PUF.

Leonardi, Paul M. (2013). "Theoretical foundations for the study of sociomateriality». Information and Organization, 23(2): 59-76.

Leonardi, Paul M. y Barley, Stephen R. (2008). "Materiality and change: challenges to building better theory about technology and organizing». Information and Organization, 18: 159-176.

Leonardi, Paul M. y Barley, Stephen R. (2010)+ «What's under construction here? Social action, materiality, and power in constructivist studies of technology and organizing». Academy of Management Annals, 4(1): 1-51.

Marx, Gary T. (2017). Windows into the soul. Chicago: Chicago University Press.

Mayntz, Renate (1989). Sociología de la Organización. Madrid: Alianza.

McCarthy, Edward (2013). "The dynamics of culture, innovation and organizational change: a nano-psychology future perspective of psycho-social 
and cultural underpinning of innovation and technology». AI E Society, 28: 471-482.

Mintzberg, Henry (1990). The structuring of organizations. A synthesis of research. Englewood Cliffs: Prentice Hall.

Mohamed, Amed A. y Gardner, William L. (2004). «An exploratory study of interorganizational defamation. An organizational impression management perspective». Organizational Analysis, 12:129-145.

Mohammadmoradi, A.; Ashrafi, H. y Fakhar R. (2014). «Impression management for the benefit of the organization or individual». Indian Journal of Fundamental and Applied Life Sciences, 4(1): 1262-1297.

Morente, Fran (2017). Las estructuras habitadas. Ideocultura y praxis de la innovación. Departament d'Economia i Empresa de la Universitat de Vic, Barcelona (Tesis doctoral).

Morrill, Calvin (2008). "Culture and organization theory». Annals of the American of Political and Social Science, 619(1): 15-40.

Nizet, Jean y Rigaux, Nicolas (2014), La sociologie d'Erving Goffman. Paris: La Découverte.

Oldenburg, Ray (1989). The great good places. New York: Paragon House.

Parfit, Derek (1986). Reasons E Persons. Oxford: Oxford University Press.

Pinch, Trevor (2008), «Technology and institutions* living in a material world». Theory and Society, 37:461-483.

Pinch, Trevor (2010). «The invisible technologies of Goffman's sociology. From the merry-go-round to the internet». Technology E Culture, 50: 409-424.

Pollach, Irene y Kerbler, Eva (2011). "Appearing competent: a study of impression management in US and European CEO Profiles». Journal of Business Communication, 48(4): 355-372.

Powers, Clark (1981). «Role-imposition or role-improvisation. Some theoretical principles». Economic and Social Review, 14(4)ः 287-299.

Raghuram, Sumita (2013). «Identities on call; impact of impression management on Indian call centre agents». Human Relations, 66(11): 1471 1496.

Ralston, Sarah M.y Kirwood, William G. (1999). «The trouble with applicant impression management». Journal of Business and Technical Communication, 13: 190-207.

Richey, Michelle; Ravishankar, M. N. y Coupland, Christine (2016). «Exploring situationally inappropriate social media posts: an impression management perspective». Information Technology and People, 29(3): 597 617. 
SAcks, Harvey (2006). Lectures on conversation. Vol. I E II. Oxford: Blackwell.

Sallot, Lynne M. (2002). «What the public thinks about public relations: an impression management experiment»+ Journalism $\mathcal{E}$ Mass Communication Quarterly, 79(1):450-164.

Shulman, David (2007). From hire to liar. The role of deception in the workplace. Ithaca: Cornell University.

Soran, Semih y Balkan, M. Onur (2013). "The effects of impression management tactics on emotional expressions: research on banking sector». Journal of Global Strategic Management, 7(1): 154-165.

Stake, Robert (1995). The art of case study research. Thousand Oaks: Sage.

Strauss, Anselm y Corbin, Juliet (1998). Basic qualitative research. Techniques and procedures for developing grounded theory. Thousand Oaks; Sage.

TAYlor, Steven J. y Bogdan, Robert (1987). Introducción a los métodos cualitativos de investigación. La búsqueda de significados. Barcelona: Paidós.

Tidd, Joe; Bessant, John y Pavitt, Keith (2011). Managing innovation. Integrating technological, market and organizational change. New York: Wiley.

Tournier, Michel (2004). Journal Extime. Paris: Gallimard.

Turnley, William H. y Bolino, Mark C. (2001). «Achieving desired images while avoiding undesired images: exploring the role of self-monitoring in impression management». Journal of Applied Psychology, 86:351-360.

Valenzuela, Hugo; Reygadas, Luis y Cruces, Francisco (2015). «Mi trabajo es mi vida. La incrustación de los mundos de la vida y del trabajo en empresas españolas». Revista Española de Investigaciones Sociológicas, 150: 191-210.

Van MaAnen, John y Barley, Stephen R. (1985). «Cultural organization. Fragments of a theory». En: P. J. Frost, L. Moore, M. R. Louis, C. C. Lundberg. Y J. Martin (eds.). Organizational Culture. Beverly Hills: Sage.

Wägar, Karolina (2012). «Exploring the mundane and complex. The use of ethnography for studying customer-oriented learning». Qualitative Market Research, 15(2): 165-187.

WALdvogel, Jörg (2007). «Greeting and closings in workplace email»+Journal of Computer-Mediated Communication, 12: 456-477.

Wang, Catherine L. y Aнmed, Pervaiz K. (2002). «The informal structure. Hidden energies within an organization». Wolverhampton Business School Working Paper Series 2002, University of Wolverhampton.

Wayne, Sandy J.y Liden, Robert C. (1995). «Effects of impression management on performance ratings: a longitudinal study». Academy of Management Journal, 38: 232-260. 
Webb, Eugene J.; Campbell, Donald; Schwartz, Richard y Sechrest, Lee (1966). Unobstrusive reseach measures: non-reactive research in the social sciences. Chicago: University of Chicago Press.

Weick, Karl .E. (2005). Sensemaking in organizations. Thousand Oaks: Sage.

Weiss, Robert S. (1995). Learning from Strangers. The art and method of qualitative interview studies. New York: Free Press.

YIN, Robert K. (2011).Qualitative research from start to finish. New York: Guilford Press.

Zavattaro, Staci M. (2013). «Social media in public administration's future». Administration E Society, 45(2): 123-158.

Zerubavel, Eviatar (2006). The elephant in the room. Oxford: Oxford University Press. 